\title{
Trajectoires de l'agriculture familiale face aux changements climatiques dans la zone agro-écologique 3 du Bénin
}

\author{
Biaou Ibidun Hervé Chabi, Akibou A. Akindele, Hervé Yabi, and Euloge Ogouwale \\ Laboratoire Pierre PAGNEY «Climat, Eau, Ecosystèmes et Développement »(LACEEDE), \\ Abomey-Calavi, BP 15 Allada, Bénin
}

Correspondence: Biaou Ibidun Hervé Chabi (chabiherv@yahoo.fr)

Published: 16 November 2021

\begin{abstract}
Résumé. La présente recherche décrit la trajectoire de l'agriculture familiale en lien avec les changements climatiques dans la Zone Agro-Ecologique 3 du Bénin (ZAE-3). Les données utilisées sont obtenues auprès des exploitants agricoles grâce à la Méthode d'Investigation Socio-Historique. L'analyse de la trajectoire de l'agriculture familiale a été faite suivant trois générations d'agriculteurs. Ainsi, la Classification Hiérarchique Ascendante et l'Analyse en Composantes Principales sont mises à contribution pour l'analyse des principales étapes d'évolution des exploitations agricoles familiales (EAF). Face aux effets des changements climatiques, les exploitants agricoles ont mis en œuvre diverses mesures ayant favorisé l'adaptabilité de l'agriculture familiale. Au-delà de leurs mérites, lesdites mesures présentent de limites qui se résument à leur inefficacité face aux événements climatiques extrêmes. Des mutations et transformations (techniques et sociales) profondes sont intervenues dans le processus d'évolution de l'agriculture familiale. Ainsi, dans le cadre de l'adaptation aux changements climatiques, quatre (4) grandes étapes ont marqué l'évolution des EAF à savoir le maintien du système traditionnel de production (13\%), l'agrandissement de la taille de l'exploitation (18\%), la tendance à l'intensification et l'innovation au sein des EAF (19\%) et la diversification des activités agricoles (49\%).
\end{abstract}

\section{Introduction}

Le système climatique dans lequel s'inscrit l'Afrique de l'Ouest en général, et le Bénin en particulier, subit des modifications à grande échelle qui restent amplifiées par les facteurs naturels et anthropiques tant régionaux que locaux. Ainsi, les climats ouest africains et béninois sont sujets à de fortes variabilités ou à des changements selon les échelles de temps et d'analyse dont les conséquences restent néfastes pour le développement durable (Boko, 1988; Afouda, 1990; Ogouwalé, 2006). Pourtant, au Bénin, l'agriculture, principalement celle de type familial, repose sur de petites exploitations familiales paysannes orientées vers la polyculture. Environ $34 \%$ des exploitations couvrent moins de 1 ha (PAM, 2014).

Dans la Zone Agro-Ecologique 3 (ZAE-3) du Bénin, le caractère pluvial de l'agriculture familiale la rend vulnérable aux effets des changements climatiques. Mais, de par ses ca- ractéristiques intrinsèques, elle s'adapte plus ou moins aux changements observés. Ainsi, malgré les changements climatiques révélés par de nombreuses recherches scientifiques, les petits producteurs continuent d'exister et d'exercer leur activité de production. Cela s'explique par le fait que ceuxci ont su s'adapter aux contraintes qui s'imposent par des mesures mises en œuvre en fonction du temps. Ainsi, le contexte climatique dans lequel l'agriculture familiale a évolué, amène à se demander quelle est la trajectoire de l'agriculture familiale face aux changements climatiques dans la ZAE-3 du Bénin? La présente recherche analyse les principaux facteurs favorisant l'adaptabilité de l'agriculture familiale ainsi que les grandes étapes de son évolution face aux effets des changements climatiques.

La ZAE 3 est située entre $9^{\circ} 11^{\prime}$ et $10^{\circ} 55^{\prime}$ de latitude nord et entre $1^{\circ} 24^{\prime}$ et $3^{\circ} 47^{\prime}$ de longitude est. Cette zone est composée de huit Communes à savoir : Bembèrèkè, N'Dali, Nikki, Kalalé, Kouandé, Péhunco, Pèrèrè et Sinendé (Fig. 1). 


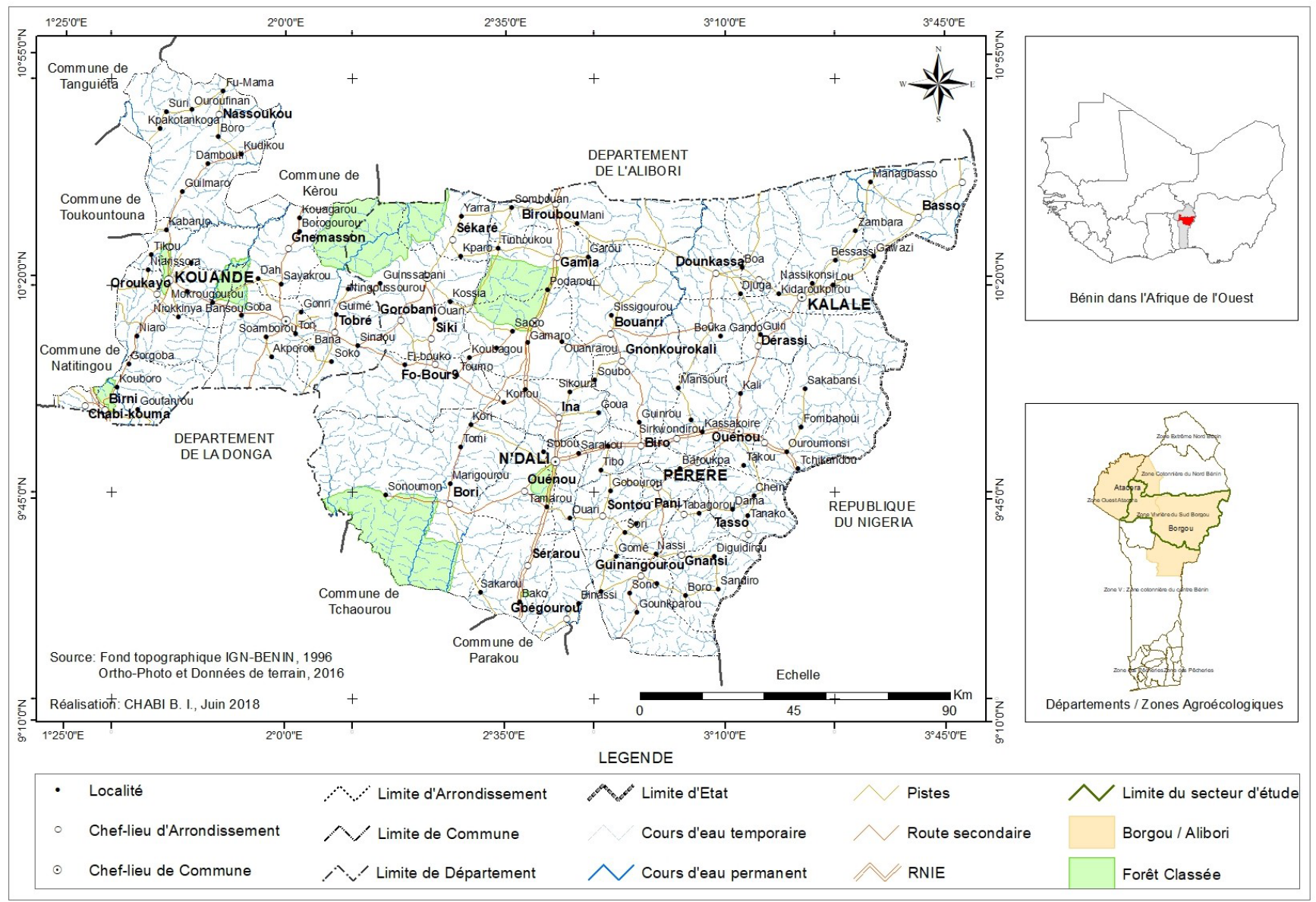

Figure 1. Situations géographique et administrative de la ZAE 3 du Bénin.

\section{Données et méthodes}

La Méthode d'Investigation Historico-Sociologique (MIHS) inspirée des travaux de Chabi et al. (2019), est utilisée pour la collecte des données sur la trajectoire de l'agriculture familiale. La période retenue pour l'analyse va de 1951 à 2015. En effet, pour apprécier la dynamique d'adaptabilité de l'agriculture familiale aux changements climatiques, la collecte de données socio-anthropologiques sur l'évolution de l'agriculture familiale a été faite auprès de trois générations d'agriculteurs. L'approche descriptive est utilisée pour l'analyse de la trajectoire de l'agriculture familiale. Celle-ci a permis de mettre l'accent sur ses caractéristiques pour mieux déterminer les facteurs de son évolution en lien avec les changements climatiques.

Ainsi, pour décrire les changements historiques intervenus dans la structure des exploitations agricoles, tout en minimisant les erreurs potentielles de données en raison de l'échec dans la mémoire des agriculteurs, les états successifs des exploitations et des ménages ont été décrits suivant des générations à l'aide d'un ensemble de variables qualitatives et quantitatives. Pour décrire l'évolution des caractéristiques des exploitations, un pas de temps de dix ans, permettant de capter les évolutions principales sur la période d'étude a été utilisé par Ryschawy et al. (2014). Contrairement à Ryschawy (2012), dans le cadre de cette analyse, le pas utilisé est relatif aux différentes périodes générationnelles retenues.

La typologie des trajectoires a été construite à partir des résultats obtenus selon une méthode d'analyse en deux étapes fréquemment utilisée pour l'élaboration des typologies (Garcia-Martínez et al., 2009) : (i) le tableau a été soumis à une analyse en composantes principales (ACP), qui a permis de retenir quatre facteurs selon le critère de Kaiser (valeurs propres $>1$ ) et (ii) les coordonnées des exploitations sur les quatre premiers facteurs de l'ACP ont été utilisées pour effectuer une Classification Ascendante Hiérarchique (CAH) selon la distance euclidienne et le critère d'agrégation de Ward. La classification a ensuite été stabilisée en utilisant la méthode des $K$-means avec les centroïdes des classes obtenues par la CAH fixés comme barycentres. Les logiciels SPSS 21 et XLSTAT v 2008 sont utilisés pour les analyses statistiques. 


\begin{tabular}{|c|c|c|c|}
\hline Parcelle $\mathrm{A}=$ & Parcelle B & Bâtiments & Parcelle C \\
\hline 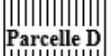 & Parcelle E⿱ E & \multicolumn{2}{|c|}{ Parcelle F } \\
\hline
\end{tabular}

Figure 2. Configuration de l'espace d'une exploitation agricole familiale.

\section{Résultats}

\subsection{Facteurs d'adaptabilité de l'agriculture familiale}

Les mesures ayant permis l'adaptation de l'agriculture familiale aux changements climatiques sont diverses. Il s'agit principalement de l'usage de la main-d'œuvre familiale et externe, la diversification de cultures, le développement des activités secondaires (égrenage de maïs, d'arachide, du riz, etc.), l'accès des producteurs aux crédits agricoles, la mutation au niveau des outils de production, l'organisation du terroir des EAF, l'association et la rotation des cultures, l'adoption des variétés à cycle court, le semis échelonnés, l'usage des herbicides, l'association agriculture-élevage et la gestion de la surface agricole utile.

\subsection{Changements techniques intervenus dans les EAF}

\subsection{Mutation au niveau des outils}

Les producteurs agricoles abandonnent progressivement la houe au profit de la charrue et des motoculteurs. En effet, aujourd'hui, la houe est moins utilisée pour le billonnage (Fig. 2).

Certes, des changements qualitatifs sont intervenus pour améliorer les outils de production. Ceux-ci visent à réduire le temps mis pour labourer les champs. Aucune machine n'est utilisée pour la confection des buttes qui reste encore plus difficile que le billonnage. La Planche 1 illustre les outils utilisés pour le billonnage.

La photo 1.1 de la planche présente un motoculteur issu de la fabrication artisanale à Bembèrèkè. Ce motoculteur est fabriqué à l'aide d'un moteur à quatre temps. Il permet aux producteurs de labourer les surfaces à ensemencer plus vite que la charrue. La charrue, en activité sur la photo 1.2 prise à N'Dali, est la plus ancienne utilisée parmi ces trois outils. Le tracteur en pleine activité de labour à Nikki (1.3) est le moyen le plus rapide. Il est accessible aux producteurs qui disposent de moyens pour en louer.

\subsubsection{Organisation du terroir des EAF d'hier à nos jours}

L'adaptation de l'agriculture familiale aux changements climatiques est favorisée par les capacités que développent les producteurs qui réajustent des mesures développées après les évènements climatiques extrêmes. En effet, depuis le dé- but des années 1970, le terroir des EAF a été morcelé en plusieurs parcelles cultivables. Ainsi, le nombre moyen de parcelles va de 2 à 6 parcelles dans le secteur. Pour les producteurs, «plus on pratique plusieurs cultures à la fois, plus on a la chance d'avoir un peu de récolte de chacune d'elles et moins on est vulnérable aux conséquences du raccourcissement de la durée de la saison culturale ». Aussi, dans l'organisation actuelle des EAF, une parcelle est réservée pour servir de zone de pâture aux animaux de trait (Fig. 3). Celleci est indirectement mise en jachère de courte durée.

Pour plus d'efficacité dans le fonctionnement des EAF, il a été mis en place un système d'association et de rotation des cultures sur l'exploitation. L'association a permis la diversification culturale. Tout ceci est favorisé par le système d'assolement (morcèlement de l'espace agricole en des parcelles).

\section{4 Étapes d'évolution passées des exploitations agricoles familiales}

L'agriculture familiale s'est profondément transformée au cours de son parcours d'adaptation aux changements climatiques. Cette métamorphose amène à dire que l'on est passé d'une agriculture paysanne traditionnelle à celle familiale. Face aux contraintes imposées par les changements climatiques en cours, les mesures d'adaptation autonomes et institutionnelles mises en œuvre par les agriculteurs, rendent compte de la diversité des trajectoires de l'agriculture familiale. Aussi, la complexité de l'organisation des EAF dépendelle des étapes de trajectoire. La Fig. 4 présente les principaux types de trajectoire (T1-T4) de l'agriculture familiale identifiés après l'analyse des données.

Concernant le premier type de trajectoire, il faut noter que les EAF de cette catégorie ont conservé les pratiques traditionnelles de production surtout en ce qui concerne la population exploitante. Il s'agit des EAF très faiblement orientées vers le marché. Elles estiment que pour durer dans le temps et résister aux menaces climatiques, «il faut être nombreux à travailler ensemble pour constituer une force de production ». Par conséquent, face aux changements climatiques, la mobilisation de la main-d'œuvre familiale s'impose. Cette dernière est essentiellement utilisée dans ces exploitations pour les opérations culturales.

Le deuxième type de trajectoire est basé sur l'extension des superficies. Beaucoup d'exploitations ont opté pour cette mesure pour faire face aux changements climatiques. Il s'agit des EAF plus ou moins orientées vers le marché. Selon ces exploitants, «pour satisfaire les besoins de la famille et avoir des revenus agricoles pour investir dans l'exploitation, la diversification des activités est nécessaire ».

La diversification culturale et celle des sources de revenus est basée sur l'augmentation ou non des superficies. Pour mieux s'adapter aux effets des changements climatiques, les agriculteurs vont opter pour la valorisation de plusieurs cultures sur la même exploitation. Pour ces derniers, «plus 

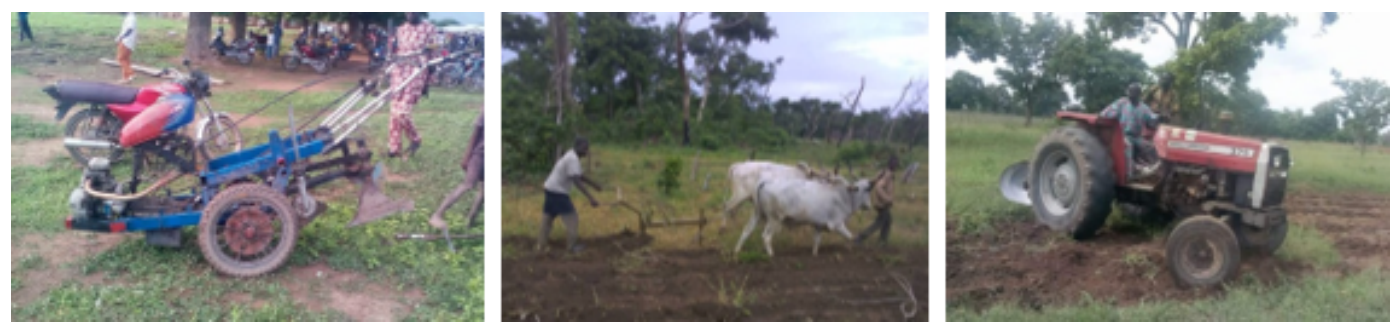

Plate 1. Outils les plus utilisés pour les opérations de labour.

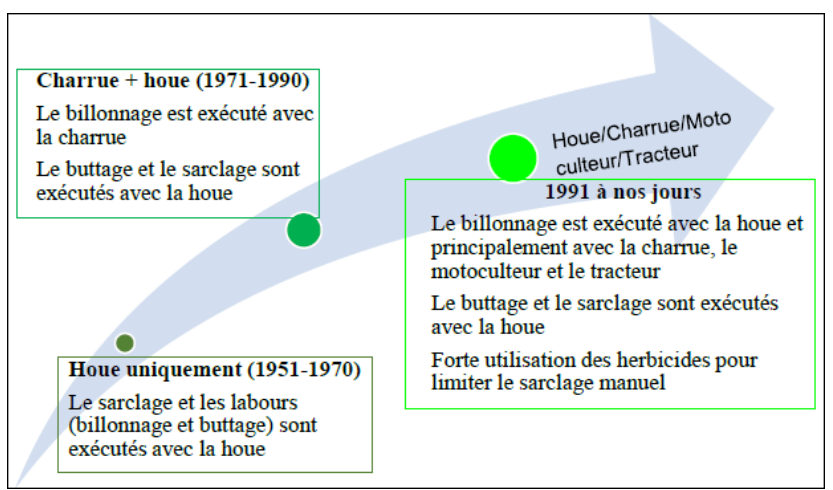

Figure 3. Changements intervenus dans l'outillage de la production agricole.

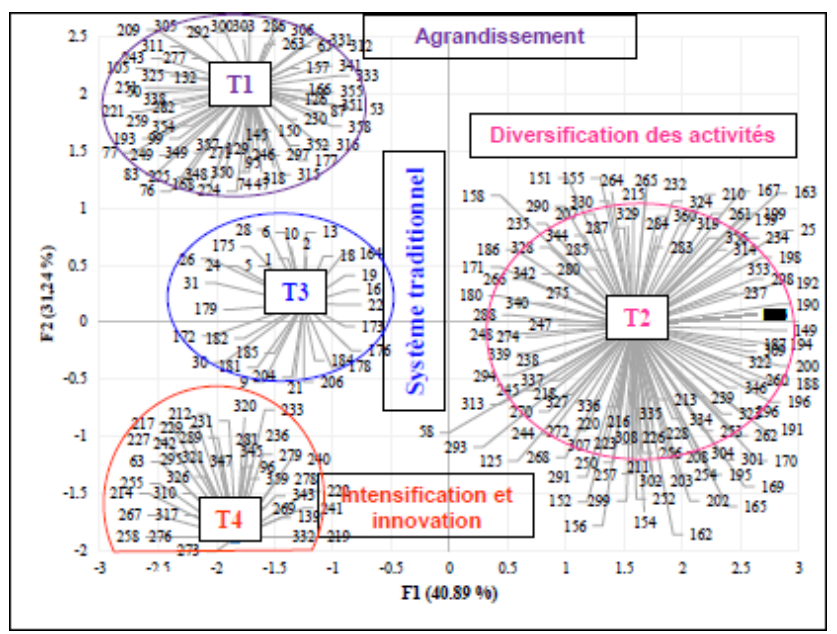

Figure 4. Typologie des trajectoires de l'agriculture familiale dans la ZAE-3.

les cultures sont nombreuses, moins on est vulnérable et plus on a la chance de récolter une petite quantité au moins au niveau de chacune des cultures $»$.

Le quatrième type de trajectoire est fondé sur l'intensification agricole et l'innovation. L'agriculture familiale est en ses débuts d'intensification. Pour améliorer les rendements culturaux, les agriculteurs ont apporté des innovations telles que l'association agriculture-élevage, l'usage des in- trants chimiques (engrais et pesticides), l'utilisation des semences améliorées à cycle court, la faible adoption de l'association des cultures.

Les différentes analyses permettent de caractériser chaque génération par des qualificatifs propres du point de vue de la technicité de l'activité de production agricole. Ainsi, on a la « génération de culture manuelle», la "génération de culture attelée » et la «génération de culture motorisée ».

\section{Discussion des résultats}

La présente étude sur la trajectoire de l'agriculture familiale face aux changements climatiques a identifié quatre principaux chemins empruntés par les exploitations. Il s'agit de l'agrandissement des superficies, la diversification culturale, le maintien du système traditionnel et l'intensification et l'innovation. La méthode utilisée est identique à la démarche suivie par Ryschawy et al. (2013, 2014) et Ryschawy (2012). Ainsi, Ryschawy et al. (2013) ont analysé la diversité des trajectoires agricoles entre 1950 et 2005 dans les Coteaux de Gascogne afin d'identifier la diversité des parcours qui ont permis la survie des systèmes de polyculture-élevage et les forces motrices de cette survie. Les modèles individuels de trajectoire d'exploitation ont été réalisés par Ryschawy (2012) avec l'usage de 20 indicateurs pour l'étude des différences d'évolution des exploitations.

Plusieurs travaux similaires ont été réalisés sur les trajectoires et la dynamique des exploitations agricoles en particulier les exploitations d'élevage en Europe (Brédart et Stassart, 2017; Ruef et al., 2012; Ryschawy et al., 2013; Vall et al., 2017; Garcia-Martínez et al., 2009; Moulin et al., 2008). Ainsi, au terme de leurs travaux sur les trajectoires passées d'exploitations dans les coteaux de Gascogne, Ryschawy et al. (2014) identifient cinq types de trajectoire d'évolution. Selon ces derniers, pour durer dans le temps, les chemins empruntés ont consisté (i) à rechercher la maximisation de l'autonomie par une coordination forte entre culture et élevage au sein de l'exploitation; (ii) à la recherche permanente de l'innovation technologique; (iii) à la diversification des ateliers de production agricole pour bénéficier d'économies de gamme et se sécuriser par rapport aux fluctuations du marché des intrants et des produits agricoles; (iv) à la sécurisation de l'exploitation par l'acquisition de capital via l'agran- 
dissement et (v) aux adaptations progressives de pratiques «traditionnelles » en lien avec la main-d'œuvre familiale disponible.

De même, les travaux de Rueff et al. (2012) sur le changement de trajectoires des exploitations familiales depuis 1950 dans la région des montagnes des Pyrénées a identifié quatre (4) types de trajectoire agricole. Il s'agit du maintien au système traditionnel, la diversification des activités au sein de l'exploitation, la diversification des activités non agricoles et la spécialisation.

\section{Conclusion}

L'adaptabilité de l'agriculture familiale aux changements climatiques est due à la complémentarité des différentes mesures d'adaptation qui sont en grande partie tant autonomes qu'institutionnelles. L'utilisation des outils modernes et semi-modernes a révolutionné l'agriculture familiale. Face aux changements climatiques, elle fait partie des formes d'agriculture les plus adaptées. Malgré son niveau d'adaptabilité aux changements climatiques, elle est toujours dépendante des facteurs climatiques et susceptible d'être vulnérable à la survenance des phénomènes météorologiques extrêmes. Au-delà des changements climatiques, d'autres facteurs non climatiques ont contribué aussi à l'évolution de l'agriculture familiale. Les différentes tendances de trajectoire passée des exploitations agricoles ont permis d'adapter l'agriculture familiale aux effets des changements climatiques. Mais, il faut reconnaître que chaque type de trajectoire présente des limites.

Disponibilité du code. La publication d'un ouvrage vise pour objectif de le rendre accessible au grand public. A partir de cet instant, le document est accessible à tous sans condition aucune. Ainsi, je déclare que le manuscrit soit accessible à ceux qui le désirent.

Disponibilité des données. Je déclare que le manuscrit soit accessible à ceux qui le désirent. Les données utilisées dans ce texte proviennent de nos propres recherches.

Collaborateurs. $\mathrm{BIHCH}$ is the main author and initiator of the research. AAA is a co-author and he participated in the collection and processing of data. HY is a co-author and he participated in the data collection. EO is a co-author and he directed the work and made the internal evaluation of the article.

Intérêts concurrents. Les auteurs déclarent qu'ils n'ont aucun conflit d'intérêts.
Clause de non-responsabilité. Publisher's note : Copernicus Publications remains neutral with regard to jurisdictional claims in published maps and institutional affiliations.

Déclaration du numéro spécial. This article is part of the special issue "Hydrology of Large River Basins of Africa". It is a result of the 4th International Conference on the "Hydrology of the Great Rivers of Africa", Cotonou, Benin, 13-20 November 2021.

Remerciements. Nous remercions tous ceux qui ont contribué de quelconque manière à la réalisation de cet article. En particulier, nous témoignons nos reconnaissances aux agriculteurs qui ont favorisé la collecte des données lors de nos investigations.

\section{Références}

Afouda, F. : L'eau et les cultures dans le Bénin central et septentrional : étude de la variabilité des bilans de l'eau dans leurs relations avec le milieu rural de la savane africaine, Thèse de Doctorat nouveau régime, Institut de géographie, Université de Paris IV, Sorbonne, p. 428, 1990.

Boko, M. : Climats et communautés rurales du Bénin : Rythmes climatiques et rythmes de développement, Thèse de doctorat d'Etat ès Lettres et Sciences Humaines, 2 volumes, CRC, URA 909 du CNRS, Université de Bourgogne, Dijon, p. 601, 1988.

Brédart, D. and Stassart, P. M. : When farmers learn through dia$\log$ with their practices : A proposal for a theory of action for agricultural trajectories, J. Rural Stud., 53, 1-13, 2017.

Chabi, B. I. H., Akindélé, A. A., et Ogouwalé, E. : Dynamique d'adaptabilité de l'agriculture familiale aux changements climatiques dans la zone agro-écologique 3 du Bénin, 3ème année, no. 5, Vol. III, Baluki, Yolande, Berton-Ofoueme, 382-397, 2019.

Garcia-Martínez A., Olaizola, A., and Bernués, A. : Trajectories of evolution and drivers of change in European mountain cattle farming systems, Animal, 3, 152-165, 2009.

Moulin, C.-H., Ingrand, S., Lasseur, J., Madelrieux, S., Napoléone, M., Pluvinage, J., et Thénard, V. : Comprendre et analyser les changements d'organisation et de conduite de l'élevage dans un ensemble d'exploitations : propositions méthodologiques, in : L'élevage en mouvement : flexibilité et adaptation des exploitations d'herbivores, édité par : Dedieu, B., Chia, E., Leclerc, B., et Moulin, C.-H., Éditions Quae, Paris, 181-196, 2008.

Ogouwalé, E. : Changements climatiques dans le Bénin méridional et central : Indicateur, scénario et prospectives de la sécurité alimentaire, Thèse de doctorat unique, EDP/FLASH, UAC, Abomey-Calavi, p. 302, 2006.

PAM - Programme Alimentaire Mondial : Analyse Globale de la Vulnérabilité et de la Sécurité Alimentaire (AGVSA), République du Bénin, PAM Bénin/Togo, Cotonou, p. 146, 2014.

Rueff, C., Choisis, J.-P., Balent, G., and Gibon, A. : A Preliminary Assessment of the Local Diversity of Family Farms Change Trajectories Since 1950 in a Pyrenees Mountains Area, J. Sustain. Agricult., 36, 564-590, 2012. 
Ryschawy, J. : Eclairer les conditions de maintien d'exploitations de polycultureélevage durables en zone défavorisée simple européenne, Une étude de cas dans les coteaux de Gascogne, Thèse de doctorat, INP-Université de Toulouse, Toulouse, 2012.

Ryschawy, J. Choisis, N., Choisis, J. P., and Gibon, A. : Paths to last in mixed crop-livestock farming : lessons from an assessment of farm trajectories of change, Animal, 7, 673-681, 2013.
Ryschawy, J., Choisis, N., Choisis, J.-P., Joannon, A., et Gibon A. : Quelles stratégies pour un maintien de la polyculture-élevage? Une étude des trajectoires passées d'exploitations dans les coteaux de Gascogne in L'agriculture en famille : travailler, réinventer, transmettre, Edp sciences, France, 287-304, 2014.

Vall, E., Marre-Cast, L., et Kamgang, H. J. : Chemins d'intensification et durabilité des exploitations de polycultureélevage en Afrique subsaharienne : Contribution de l'association agriculture-élevage, Cah. Agric., 26, 25-36, 2017. 